$\frac{\text { RHODES UNIVERSITY }}{\text { Where leaders learn }}$
Psychology Department

1 University Road, Grahamstown, 6139, South Africa PO Box 94, Grahamstown, 6140, South Africa

$\mathrm{t}:+27(0) 466038500$

f: +27 (0) 466037614

e: psychology@ ru.ac.za

www.ru.ac.za/psychology

\title{
Keeping a lid on your mental health during the lockdown
}

These are extraordinary times that warrant unprecedented measures. Most of us are frightened about becoming ill and dying, losing loved ones, losing income, losing the opportunity to complete our studies, and being separated from people that we care about for an extended period of time. Many are worried about whether or not they will be able to access course material online, or keep up with the demands without good internet access. Others are frightened that they may be deprived of life-saving medication or food. All these fears are perfectly understandable. It is important to remember that anxiety is a physiological and psychological response to threat, and the novel coronavirus disease is a very real threat. An increase in anxiety that does not become debilitating is, for many, an appropriate response to the situation, and one that will help us to ensure that we treat this threat seriously.

For many, though, the fear and stress, coupled with the disruption to our lives brought on by a necessarily strict confinement, enforced social distancing and inactivity, will exacerbate existing psychological challenges that include excessive fear, worry, sadness, rumination, panic, and obsessions and compulsions. In the absence of our usual coping mechanisms or as a result of prolonged boredom, some will drink excessively, binge on food, social media, or Netflix, or selfharm, or use other substances to regulate our moods. The guidance below is a brief compilation of suggestions that might help you better manage your mood and anxiety over the lockdown period (or help prevent these from worsening), when so many of our normal coping mechanisms are necessarily curtailed. The list starts with some general suggestions that are applicable to all of us, including those who already struggle with psychological distress, and then goes on to describe some specific suggestions for those of us who struggle with specific anxiety disorders and depression. However, this is not a substitute for psychological or psychiatric treatment. 


\section{General suggestions}

Perhaps most important is to stay in touch with people that you care about. Take advantage of digital technologies that allow you to send text messages or voice messages, to make voice and/or video calls, and to share images and photos. Loneliness and enforced social withdrawal can cause our mood to sink. WhatsApp, Skype, Facebook and other social media platforms are pretty good substitutes when we are physically separated from one another. Use these platforms to stay involved in the lives of the people you care about. Check in on loved ones, friends, and people who you know are alone or struggling.

Maintain a routine. The tendency, unless you are particularly conscientious, is that without the external anchors that are provided by lectures, work and social obligations, our normal routine will slip: Instead of a normal bedtime, we stay up late and sleep whenever we feel like it. Yet regular and consistent sleep is an important factor in maintaining good mental health. Now is the time, more so than usual, to go to bed at the same time each night and wake up at the same time in the morning. Try to ensure that your day has some purpose.

Opportunities to exercise have been seriously curtailed by the rules of the lockdown and this will have a negative impact on those who use rigorous exercise to maintain a healthy mood. It will also be difficult for those who are active or competitive sports men and women. (Lots of good research shows that exercise is a powerful antidepressant. It also helps with anxiety.) But while these opportunities are curtailed, it is still possible to get some exercise: Push-ups, sit-ups, squats and a host of other exercises can easily be done in confined spaces. There are many demonstrations on YouTube. And if you have the space, use it. Certainly, avoid spending the whole day lying or sitting down.

Manage your consumption of news. It is easy to get caught up in the endless cycle of news. Try not to check the news compulsively. If you are the sort of person that likes to keep yourself informed, which is good, then schedule news time. But do be careful of what news you consume. Stick with reputable sources. Get your information about COVID-19 from The World health Organization (WHO), Centers for Disease Control and Prevention (CDC), National Institute for Communicable Diseases (NCID) and our own National Department of Health.

Watch your drinking. Alcohol is a depressant. And not only does it interfere with the regulation of your mood, exacerbate interpersonal and other domestic problems, it is also bad for your health, 
including the health of your lungs. According to Charles Parry, Director: Alcohol, Tobacco and Other Drug Research Unit at the South African Medical Research Council, recent evidence suggests that alcohol disrupts ciliary function in upper airways, impairs function of immune cells and weakens barrier function of the epithelia in lower airways. In other words, drinking might exacerbate your risk of COVID-19 complications.

Keep taking any prescribed medication according to the prescription. Now is not a good time to change; if you do, make sure that you consult your doctor.

Nurturing the relationships with those in your household. Time during shutdown is certainly an opportunity to spend quality time with those in your household. Talk to each other about your fears and anxieties in relation to COVID-19, but also be sure to talk about your dreams for the future. If children are part of your household, include them in age appropriate conversations that allow them to express their fears and worries too. It is easy, when we are stressed and confined, for interpersonal conflict to flare up. If you find that the shutdown has resulted in an increase in conflict in your home, be sure to find time on your own when you can calm down and self soothe, before engaging in your relationships again. Perhaps develop a code word that you can use when you are feeling particularly flooded emotionally, so that everyone understands this means that you need to step away for a bit. It is particularly hard to listen and understand one another when we are emotionally activated. Caregivers of children may especially need this time out, as children will rely on you for their own emotional regulation. Remember, quality time together is more important than quantity so allow the children screen time and/or opportunities to entertain themselves so that when you spend time together you are in the right frame of mind to do so. You don't have to be the perfect caregiver or partner during these 21 days, so be kind to and forgiving of yourself and others. While the 'wheels' will 'fall off' from time to time, what is more important is that when they do, you can recover and get on track again.

2. Specific suggestions for those who struggle with obsessions and compulsions, depression, suicidal thoughts, psychological trauma, health anxiety and worry, and panic

\section{Obsessions and Compulsions}

Obsessions are intrusive thoughts, images and impulses that cause anxiety or distress, while compulsions are the mental or behavioural effort to cancel, correct, counteract or atone for the obsession. Obsessive-compulsive disorder is a problem where obsessions and resulting compulsions 
start to interfere with your social and occupational functioning. One common type of obsessivecompulsive disorder is to do with the obsessional fear of contamination and the compulsive need to wash your hands. It is of course very important to maintain personal hygiene to reduce the risk of infection with the coronavirus. But even this can be done in a way that is excessive, and possibly also counterproductive. There is already evidence that the repeated guidance to wash your hands vigorously and regularly has resulted in an increase in the severity and prevalence of obsessivecompulsive disorders. People already inclined to compulsively wash in response to the discomforting thought that they may be contaminated may find that their hand washing becomes excessive. If this has affected you and you realise that you are washing your hands to manage your anxiety rather than to manage the actual risk of infection, then try to restrict the washing (or sanitizing) of your hands to what is appropriate to manage the actual risk: you should wash your hands when you leave and return to your home, and whenever you come into contact with somebody who may be ill. At other times, resist the urge to wash your hands in response to the obsessive thought that you may be contaminated. Instead, try to sit with your discomfort until it diminishes.

Also, remember, this might not only affect those with obsessions about being contaminated and who wash their hands compulsively in response to this. The stress of the times might exacerbate any obsessions, while making your typical compulsions harder to resist.

If you are unable to keep your obsessions and compulsion in check, then be sure to seek psychological treatment after the lockdown. The evidence suggests that the most effective psychological treatments for OCD is Cognitive-Behavioural Therapy.

\section{Depression}

Social withdrawal and reduced activities are two of a number of factors that can exacerbate and maintain a depressed mood. We have already discussed how important it is that you maintain your social contact with others as far as possible, without putting yourself or anybody else at risk. This means that we should use smartphones and digital technologies to send and receive messages, to see each other and to speak with each other.

Also important is that you keep yourself active. If you are struggling with this, then use a calendar and record each of your activities and indicate on a scale of 0 to 5 how pleasurable each one is and the sense of mastery that you feel after the activity. So that is two scores, one for pleasure and the 
other for mastery, starting from 0 to indicate no pleasure or sense of mastery to 5 that indicates complete pleasure and mastery. (While some activities might be both pleasurable and give you a sense of mastery, others might be one or the other. This doesn't matter; all you must do is ensure that you are getting pleasure and mastery over different activities.) Do this for a couple of days, then review your diary. You might be able to identify activities that are good for you and those that you should keep to a minimum. If you can't think of any activities that might be more pleasurable or give you a sense of mastery, then pick and try any of these: cook a meal, clean, fix something, read a book, do some exercise, contact a friend, and so on.

\section{Suicide}

It is not uncommon to have thoughts of killing yourself when you are depressed or have some other psychological problem. While this is obviously very distressing, it is not a psychiatric emergency unless these thoughts develop into concrete plans to end your life. Research shows that many people who survive an attempt at suicide or who are prevented from killing themselves, are often relieved to have survived and do not necessarily experience further thoughts of killing themselves. Suicide is not an inevitable consequence of prolonged distress. If you reach the point where you think that you may be a danger to yourself, then reach out. Speak to a friend or family member. Or call lifeline at 0861322322.

\section{Trauma}

Two things can happen here. The coronavirus pandemic will be traumatic for some people. Health practitioners are probably most at risk, but others might be exposed to the traumatic events to do with the pandemic. The lockdown might exacerbate our already high rates of domestic violence. The other thing that may happen is that people will find that the lockdown period and continual exposure to distressing news will exacerbate their pre-existing posttraumatic stress.

There are several known risk factors that increase the likelihood of an adverse response to trauma. The nature of the traumatic event or events is an important one: the more violent the event, the more traumatic it is. Interpersonal violence carries a high risk of traumatic stress. Other important factors include people's social support before, during and after the traumatic events, experiences of previous traumas, and the level of stress that people are under at the time of the trauma.

Important to note is that psychological distress is a normal response to a traumatic event. Psychological reactions can include any of the following: Shock, denial, disbelief, anger, irritability, 
mood swings, guilt, shame, self-blame, sadness, hopelessness, anxiety, fear, social withdrawal, disconnected and/or numb. Other responses can include difficulty falling or staying asleep, upsetting dreams, being startled easily, racing heart, aches and pains, fatigue, concentration difficulties, edginess, agitation and muscle tension. People might experience involuntary distressing memories of the traumatic event. People often actively try to avoid these thoughts as well as external reminders of the events, especially the people and places associated with their traumatic experiences.

For those of you newly traumatised, give it time. Compulsory debriefing is not advisable. Trauma and stressor-related disorders describe the situations when people don't recover fully from traumatic events. In such situations, people often benefit from psychological therapy. Guidelines suggest that if you continue to experience these symptoms four or five weeks after the traumatic event, then it is best to seek psychological assistance. If after a month, it is clear that you are still struggling, then see a psychologist (many will continue to offer their services by video-conferencing technology).

\section{Health Anxiety and Worry}

Many of us worry about our health or worry that all our aspiration and dreams will be shattered by a dreaded disease and a premature death. People with serious underlying health conditions that have been identified as risk factors for a more serious case of COVID-19 should stay in isolation as long as necessary. In such cases, some worry may be unavoidable. Even then, the risk of dying of COVID-19 remains relatively low. Others, who are not at extra risk may still worry. If this worry becomes debilitating and disproportionate, you may have a problem with health anxiety.

Health anxiety is an intense worry about your health, usually to the point where it makes you feel very distressed and interferes with day-to-day living. You may be convinced you have a serious illness, even though your doctor hasn't diagnosed one. Once you start feeling anxious about your health, it can seem very difficult to stop worrying. People who are worried start to check their bodies, might seek continual reassurance from others, or search for reassuring information on the internet. They will notice and misinterpret ordinary bodily symptoms. This results in a vicious circle as the strategies we use to cope might also exacerbate our anxiety. Obviously if you have flu-like symptoms, seek medical guidance. But if you don't but still find that you are worrying excessively, resist the urge to seek reassurance, don't look for information about COVID-19 on the internet, and 
distract yourself. The more anxious you are, the more likely all your dreaded scenarios will seem. If this persists, then you may need to consult a psychologist.

If overwhelmed by worries, then try to schedule a worry period. Set aside a 30- or 45-minute period of time each day when you are allowed to let your worries run. Outside of that period you should try to postpone your worry. Instead of trying not to worry, tell yourself that you can think about this later. However, even if you have decided earlier to worry about something and arrive at your worry time to find that the worry is not as pressing as it was before, then don't use your worry period. If the worry is pressing, then let it run its course, but stop when your time is up. If you still feel like worrying, then you can postpone it to the worry period scheduled for the following day. If you struggle to let the worry go, use distraction. The important thing here is to practise; it will be hard at the start, but you will find it easier to postpone your worries the more you practice. If you keep this up, you will learn that you have much more control over your worry than you previously believed.

If you do become infected with the coronavirus, as many will, follow your medical guidance. Unless you experience very mild symptoms, this will inevitably be an anxious experience. Remind yourself that the overwhelming majority of those who become infected will recover.

\section{Panic attacks}

How do you know if you're having a panic attack? Panic attacks have four characteristics:

1. During panic attacks you experience intense fear or terror.

2. The attack comes on suddenly, often with little warning.

3. The very intense feelings tend to pass quite quickly, often within 5 or 10 minutes. (However, it may not feel quick when it happens, and can leave you feeling very drained and unsettled for a long time afterwards.)

4. During the attack you think that something really awful is about to happen or has already started to happen. You may think you're having a heart attack, or are about to suffocate. Alternatively, you may think you are going to faint, vomit, go crazy, make a fool of yourself, or lose control of your bowels.

If your worries spill over into full-blown panic attacks, then you might find a controlled breathing exercise to be helpful. One of the things that is often implicated in the escalation of anxiety into a panic attack is that we hyperventilate. Try this and practice many times each day: 
Either sit upright or lie down on your back. If possible, breathe through your nose in a gentle, steady rhythm. Your breathing should not be jerky and you should try not to gulp or gasp.

1. Place one hand on your chest and one hand on your stomach.

2. As you breathe in through your nose, allow your stomach to swell. This means that you're using your lungs fully. Try to keep the movement in your upper chest to a minimum and keep the movement gentle. Imagine that you have four lungs: two in your chest and two in your stomach area. Imagine the 'lungs' in your stomach filling up with air.

3. Slowly and evenly, breathe out through your nose. Now imagine the 'lungs' in your stomach area deflating.

4. Repeat this to establish a gentle rhythm. You're aiming to take 8 to 12 breaths a minute. This means that it should take around 5 to 7 seconds for each in-breath and out-breath cycle. But don't worry too much about the timing - you'll find a comfortable rhythm that's right for you.

5. Try to relax your mind too. Shut your eyes and concentrate on pleasant, peaceful thoughts. Feel the tensions ease in your body.

There will be opportunities to restore yourselves, to grieve our losses, and to pick up the pieces to carry on with our lives. If the best you can do is to survive this period of uncertainty and confinement, then that is good enough.

Department of Psychology

Rhodes University 